\title{
The measurement of the energy spectrum of Mrk421 by LHAASO-WCDA: MC study
}

\author{
Jiali Liu ${ }^{* \dagger}$ \\ the school of Physics science and technology, Kunming University, Kunming, China, 650214. \\ Liujiali2017d 126.com
}

for the LHAASO Collobaration

WCDA is a main component of LHAASO experiment, to measure the primary energy spectrum of gamma ray source is its main topic. The unfolding method is a traditional way in the study for the weak energy reconstructed ability of the ground array. In this paper, firstly, we estimate the significance of gamma ray signal from Mrk421 in one year. Then based on the minimum $\chi^{2}$ method, we study the detected ability of the spectrum of Mrk421 of WCDA at different observation times.

36th International Cosmic Ray Conference -ICRC2019-

July 24th - August 1st, 2019

Madison, WI, U.S.A.

*Speaker.

${ }^{\dagger}$ Supported by the NSFC through the Grant of No. 11563004. 


\section{Introduction}

Blazar is one category of Active Galaxy Nuclear (AGN), which has strong emits at all wavelengths. The most observed extragalactic sources are Blazars. The Spectral Energy Distribution (SED) of these objects exhibits double-hump structure [四]. The first one hump, around the soft to medium X-ray range, is thought to be due to synchrotron radiation from high energy electrons [[]]. The origin of the second hump ( $\mathrm{GeV} / \mathrm{TeV}$ hump) is unclear. The composition of these jets is also not known; it is not clear whether they are made of electron-positron plasma (lepton origin, SSC model) or electron-proton plasma (hadron origin).

Mrk 421 is one of the brightest BL Lac type of Blazar in the X-ray and UV sky and the first extragalactic source detected at $\mathrm{TeV}$ energies in 1912 [B]. From that time, Many space/baloon and ground experiments has observed this object at all wavebands. The associated analysis of these experiments at multiple wavebands identifies its common features. They are the double-hump structure, the strong correlation between $\mathrm{KeV} / \mathrm{MeV} \mathrm{X}$ ray and $\mathrm{GeV} / \mathrm{TeV}$ gamma ray wavebands, and the spectrum becoming harder at higher energies of all wavebands [ [ 9 ]. All these features are flavored with SSC model. However, several flare observations of Mrk421 indicates that the flux becomes strong only at gamma ray band and the experiment at $\mathrm{X}$ ray band do not observed the flare (i.e orphan flares). They can not be explained by SSC model.

A long-term, unbiased without seasonal and temperature, and durative stable monitoring can help to study the features of Mrk421. LHAASO-WCDA is designed to achieve this goal. The measurement of the energy spectrum of Mrk421 is the key issue in the monitoring. In this paper, we describe the LHAASO-WCDA experiment briefly at first. Then the method of the measurement of the energy spectrum of Mrk421 is described in detail. Finally the result and the summary are given.

\section{WCDA Experiment}

The Water Cherenkov Detector Array (WCDA), with large detected area of 78,000 $\mathrm{m}^{2}$, constituted by 3 separate arrays (see Fig. 四), is a main component of LHAASO experiment [ []]. Each array is a single water pond with $4.5 \mathrm{~m}$ water depth. Two of them are with an effective area of $150 \times 150 \mathrm{~m}^{2}$ and each pond contains 900 detected units. The third pond is with an area of $300 \times 110 \mathrm{~m}^{2}$ and contains 1320 detected units. Each unit covers a area of $5 \times 5 \mathrm{~m}^{2}$ and is divided by black plastic curtains vertically hanged in the water to avoid scattered lights from the adjacent units. The curtains of the cells are made in the black plastic to minimise late light from the reflections. A pair of circular PMTs with 8 inches and 1.5 inches in diameter in each unit at the first $150 \times 150 \mathrm{~m}^{2}$ pond and a pair of circular PMTs with 20 inches and 3 inches in diameter in each unit of the other two ponds are anchored at the center of the cell bottom. To guarantee an attenuation length of near-ultra-violet light longer than 20 meters, a water purification system is operated.

\section{Energy Spectrum Fitting}

\subsection{The Simulation of the Signal}

Given a energy spectrum model $J(E)$ (B. 


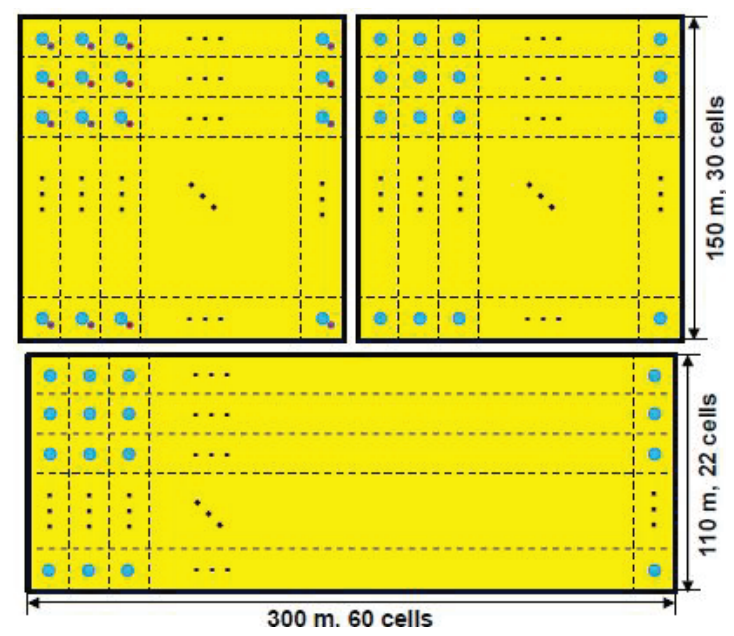

Figure 1: The WCDA layout.

by WCDA can be described as (B.2):

$$
J(E)=J_{0} E^{-\alpha} \exp \left(-E / E_{\text {cut }}\right)
$$

where $J_{0}=2.82 \times 10^{-11} \mathrm{TeV} \cdot \mathrm{s}^{-1} \cdot \mathrm{cm}^{2}, \alpha=2.21$ and $E_{\text {cut }}=5.4 \mathrm{TeV}$ [焑].

$$
N_{t o l}=\int_{E 1}^{E 2} J(E) \cdot A_{e f f} \cdot \triangle T \cdot d E
$$

where $A_{\text {eff }}$ is shown in the left panel of Fig.D, which include the effects of the detector area and the detected efficiency of WCDA experiment.
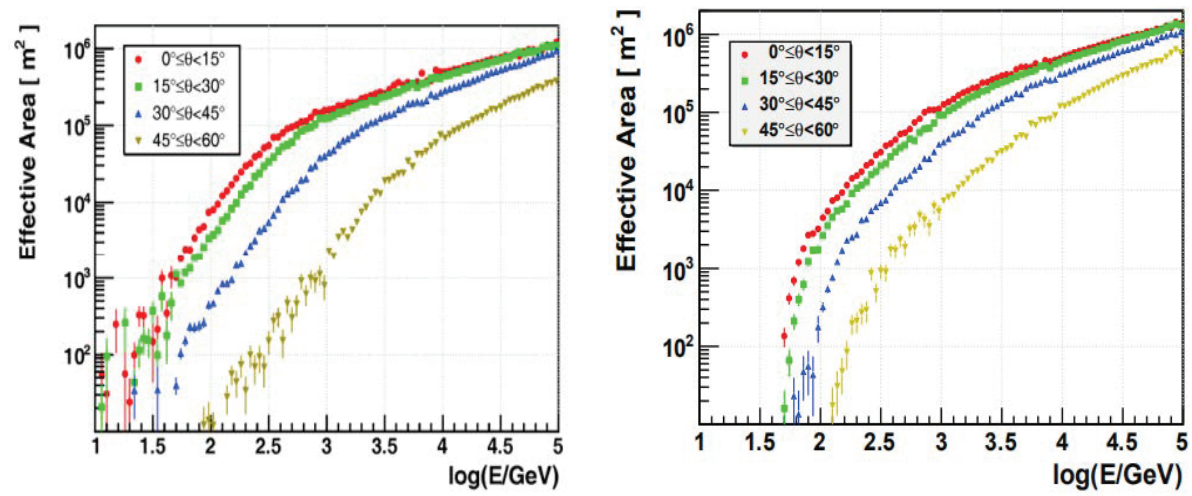

Figure 2: The distribution of WCDA $A_{\text {eff }}$ as a function of energy for gamma events (left panel) and proton events (right panel) for different zenith ranges.

$N_{t o l}$ gamma events are distributed in the equatorial coordinate based on a two-dimensional Gauss distribution according to the shower direction resolution of WCDA experiment shown in the left panel of Fig. B]. To maintain the reconstructed accuracy of the events, the events with the zenith great than $45^{\circ}$ are cut. After this cut, the observation time for each sidereal day is about 7.12 hours. The energy of the shower is sampled based on the energy spectrum model described 
in formula (B. factor depending on its $\gamma$ or $P$ selected efficiency as a function of energy that shown in the right panel of Fig. B. Considering all above factors, the signal distribution of Mrk421 are obtained in the

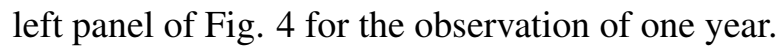
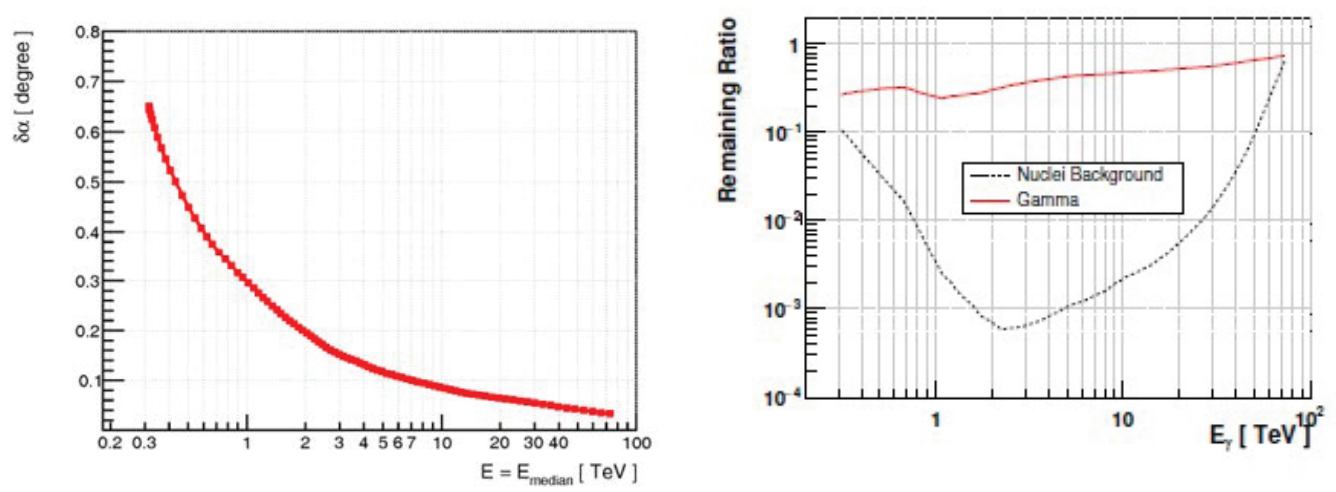

Figure 3: Left: The gamma shower direction resolution of WCDA experiment. Right: The $\gamma / P$ selected efficiency of WCDA experiment.
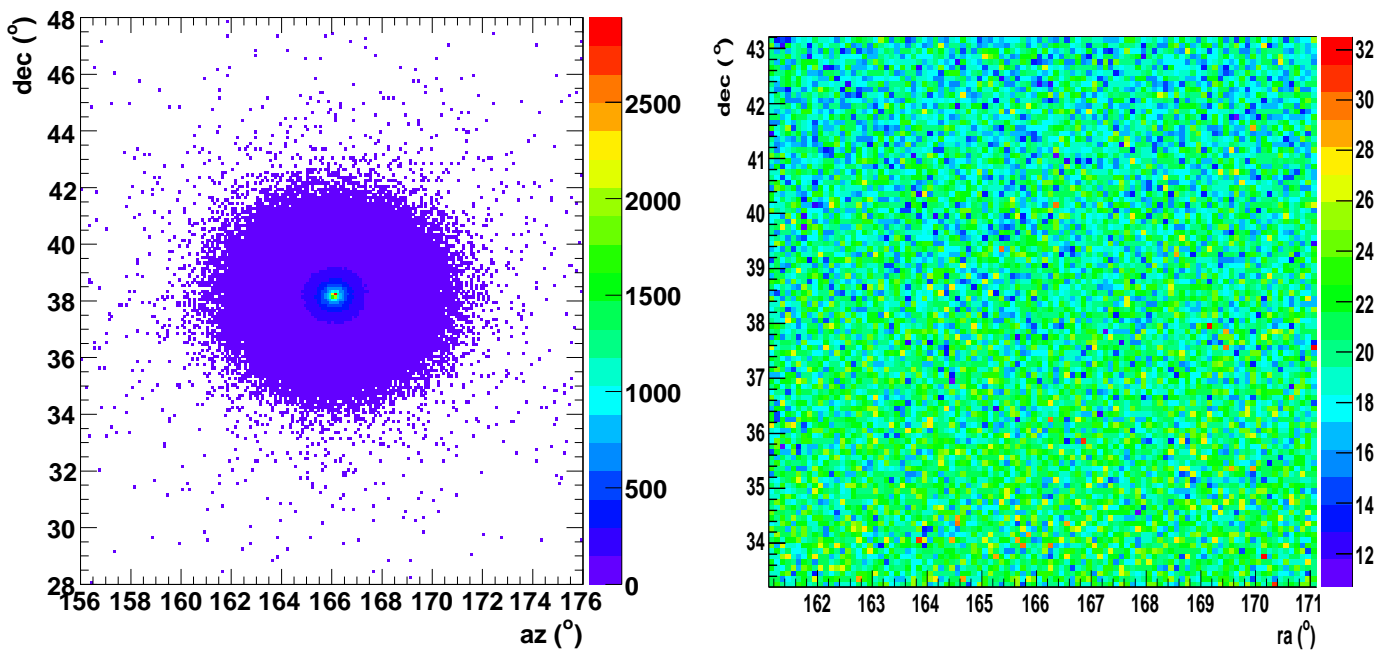

Figure 4: The signal (left) and background (right) distributions of Mrk421 in one year's observation.

For the estimation of the background, an energy spectrum of all particles according to Horandel model [ $[\mathbf{U}]$ is adopt. The effective area of WCDA for hadrons is shown in the right panel of Fig. ‥ Considering all the factors same as the gamma events, the background distribution map in one year's observation is obtained shown in the right panel of Fig. 因.

Once the signal map and background map are obtained, a significance of Mrk421 is calculated according to the Li-Ma formula [ [ $]$ ]. Considering the direction resolution of detectors, one needs to do the smoothing while calculates the significance. To get a optimal smoothing radius, a optimization procedure is done. The results are shown in the left panel of Fig. [ ] which indicates that 
the optimal smoothing radius is $0.57^{\circ}$. Under the optimal smoothing radius, the significance of Mrk421 in one year's observation are calculated and the significance map is presented in the right panel of Fig. []. The most significant point is $89.3 \sigma$.
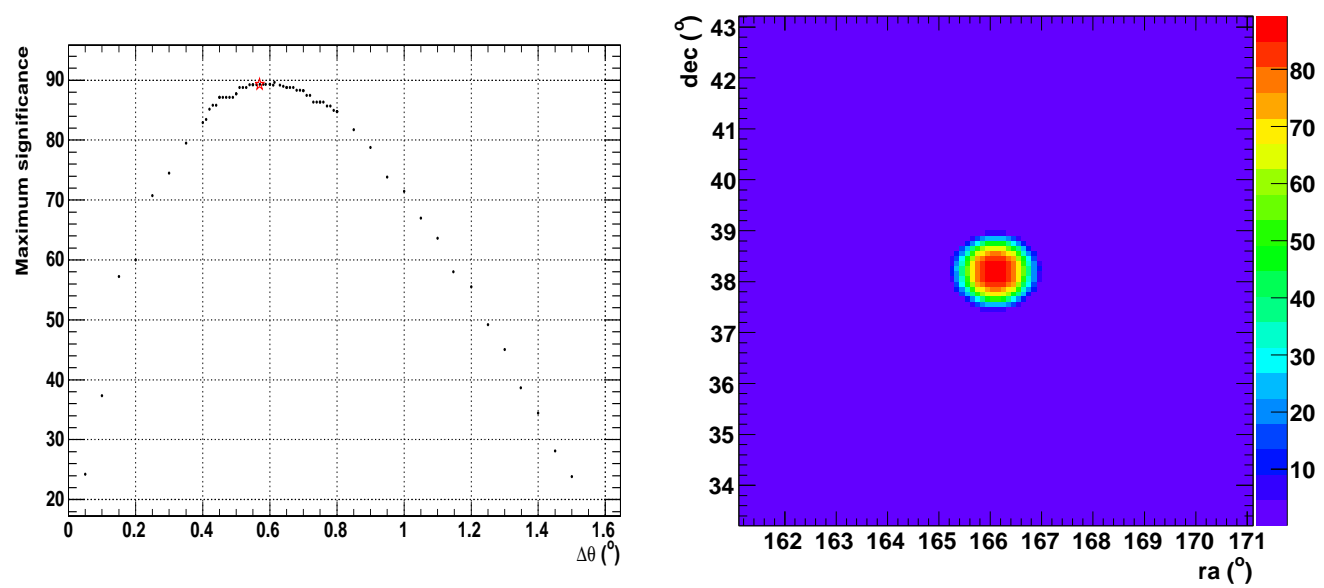

Figure 5: left: The significance distribution as a function of smoothing radius for Mrk421 in one year's observation. right: The significance of Mrk421 in one year's observation under the optimal smoothing radius.

\subsection{Mimimum $\chi^{2}$ fitting}

To obtain the primary spectrum of Mrk421, a minimum procedure by MINUIT based on the minimum $\chi^{2}$ fitting is adopted. $\chi^{2}$ function is constructed as:

$$
\chi^{2}=\sum \frac{\left(N_{\text {exp }}(i)-N_{\text {fitting }}(i)\right)^{2}}{\sigma^{2}}
$$

Here $N_{\text {exp }}(i)$ is the observed signal in $i^{\text {th }}$ pixel during a fixed observed period. $N_{\text {fitting }}(i)$ is the expected signal in the same observed period obtained as follows. For a given model (B.]) with given parameters $J_{0}, \alpha$ and $E_{c u t}$, total events $N_{t o l}$ is calculated following (B.2). Then the signal distribution (i.e. the distribution of $\left.N_{\text {fitting }}(i)\right)$ is obtained considering the geometry reconstruction accuracy and the $\gamma / P$ discrimination.

To test the measured accuracy of the Mrk421 energy spectrum, the fitting under different observed periods are carried out. Fig. B shows the gamma event distributions emitted from Mrk 421 observed by WCDA experiment for one week, one month, three months, one year and two years, respectively. For the data of less than three months, the fitting parameter is only $J_{0}$ for the statistics are not enough to do the spectrum index fitting. Fig. $\square$ shows the fitting results for one week, one month, three months, respectively. They indicate that the fitting accuracy are increased with the increase of data statistics. The fitting errors are within $\pm 10.0 \%, 4.6 \%$ and $4.0 \%$ for three observed periods, respectively.

For the data of one year and two years, both the flux $J_{0}$ and the index $\beta$ are the fitting parameters. Fig. $\mathbb{8}$ shows the fitting results. Both the fitting error for the two parameters are around $\pm 10.0 \%$. 


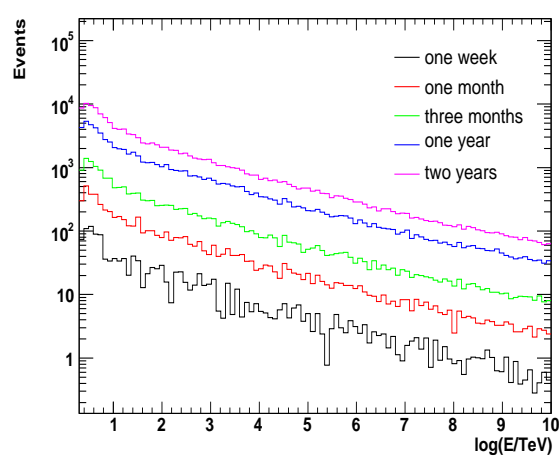

Figure 6: The event distribution as a function of energy detected by WCDA experiment at different observed time.

\section{Summary}

The measurement of the energy spectrum of point sources for WCDA experiment based on the minimum $\chi^{2}$ fitting has been carried out. Applying the procedures to Mrk421, the measured errors for the spectrum flux are within $\pm 10.0 \%, \pm 4.6 \%$ and $\pm 4.0 \%$ for the observed time of one week, one months and three months. For the measurement errors both for flux and spectrum index, they are decrease to around $\pm 10.0 \%$. In the real observation, the signals that detector measured are the PEs or the PMTs, the next work is to obtain the source spectrum with unfolding method based on Bayes analysis through these measured information.

\section{References}

[1] Fossati G., Maraschi L., Celotti A., Comastri A. and Ghisellini G., A unifying view of the spectral energy distributions of blazars, MNRAS, 1998, 299 (2): 433.

[2] Fossati, G., et al., MULTIWAVELENGTH OBSERVATIONS OF MARKARIAN 421 IN 2001 MARCH: AN UNPRECEDENTED VIEW ON THE X-RAY/TeV CORRELATED VARIABILITY, ApJ, 2008, 677: 906.

[3] Punch, M., Akerlof, C. W., Cawley, M. F., et al.,Detection of TeV photons from the active galaxy Markarian 421, Natur, 1992, 358 (6386) : 477-478.

[4] Blazejowski, M., et al., A Multiwavelength View of the TeV Blazar Markarian 421: Correlated Variability, Flaring, and Spectral Evolution, ApJ, 2005, 630(1): 130-141.

[5] Huihai He for the LHAASO Collaboration, Design of the LHAASO detectors, Radiation Detection Technology and Methods, 2018, 2:7.

[6] A. U. Abeysekara for the HAWC collabaration, Daily Monitoring of TeV Gamma-Ray Emission from Mrk 421, Mrk 501, and the Crab Nebula with HAWC, The Astrophysical Journal, 2017, 841:100.

[7] Jorg R. Horandel, On the knee in the energy spectrum of cosmic rays, Astroparticle Physics, 2003, 19: 193-220.

[8] Li, T.P., Ma, Y.Q., et al., Analysis methods for results in gamma-ray astronomy, ApJ, 1983, 272:317-324. 

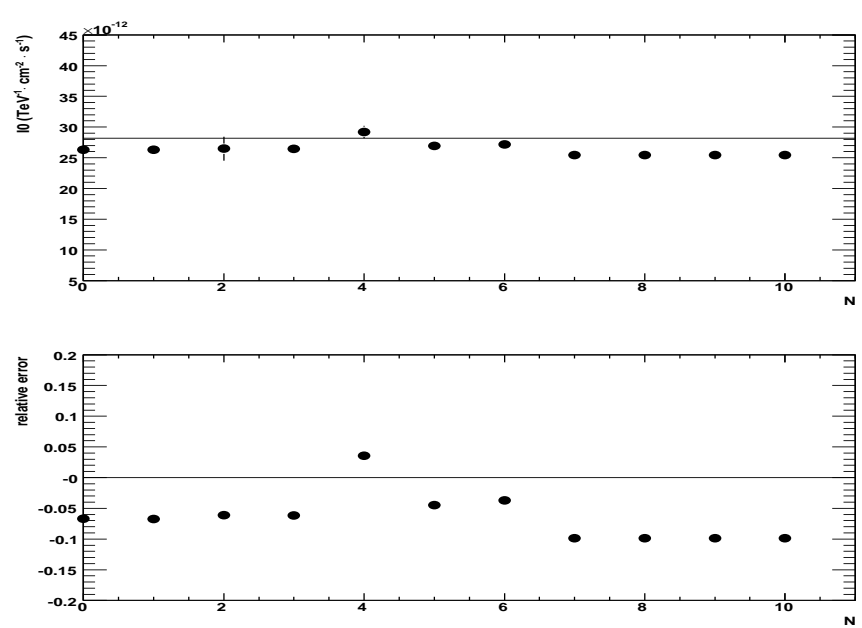

(a) one week
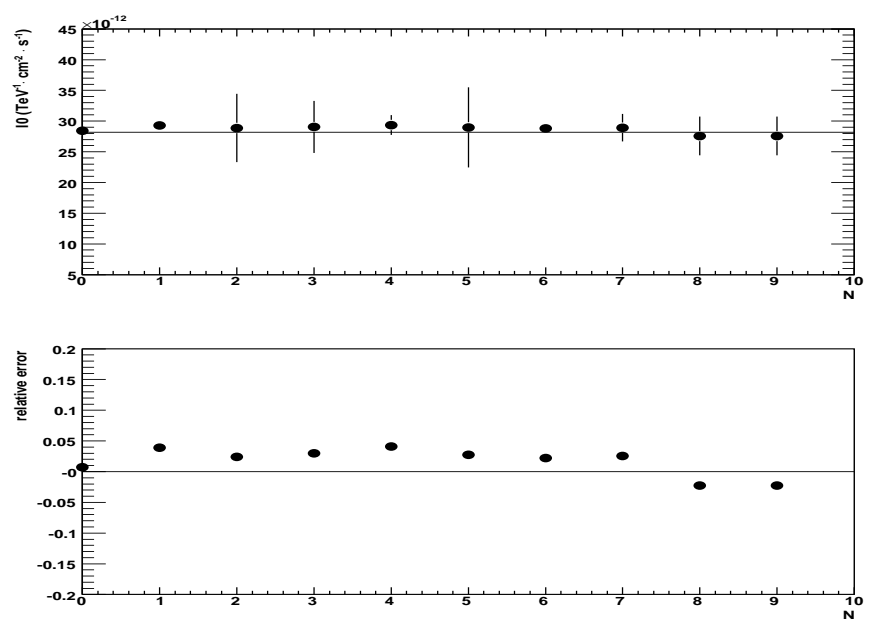

(b) one month
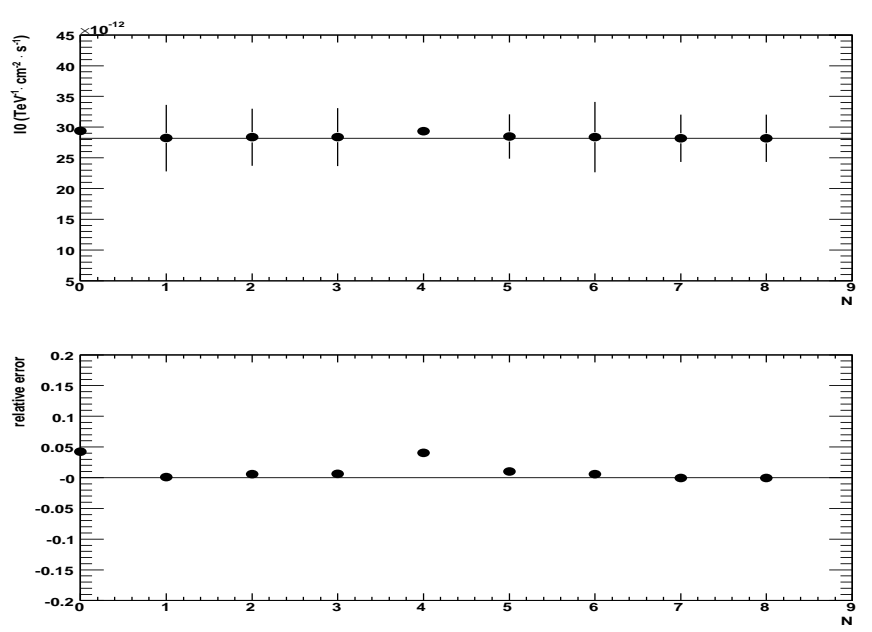

(c) three months

Figure 7: The flux fitting results (top panels) and the fitting error (bottom panels) of Mrk421 for the observed time of one week (a), one month (b) and three months (c). 

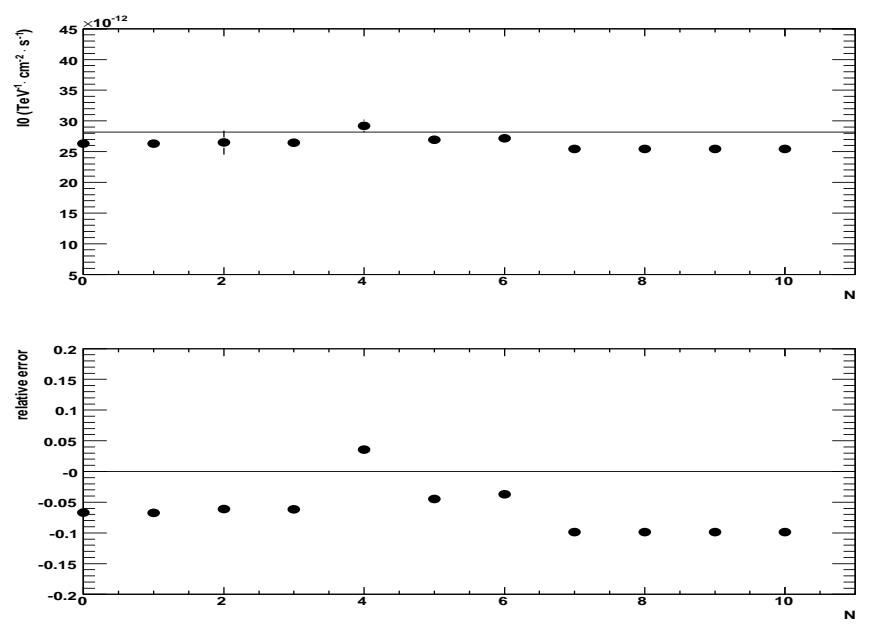

(a) $J_{0}$
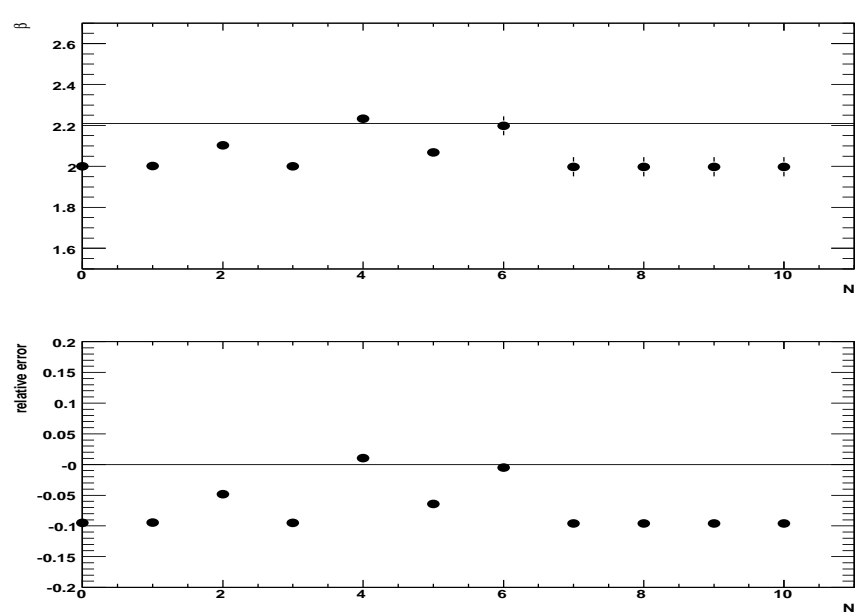

(b) $\beta$

Figure 8: The fitting results for $J_{0}$ and $\beta$ (top panels) and their fitting error (bottom panels) of Mrk421 for the observed time of one year. 